not piecemeal necrosis grade, bile duct paucity and cholangitis, or immunological laboratory data, were associated with smoking history. Multiple logistic regression analysis identified smoking intensity, years of passive smoking and significant necroinflammatory histological activity as independent risk factors of advanced liver fibrosis (F3-F4 stage) at diagnosis, adjusted for age, gender, BMI and alcohol consumption. For every pack-year increase in smoking intensity there was a 3.2 times higher likelihood of advanced fibrosis (95\% CI: 2.018-6.294).

Conclusion Our study results confirm the previously reported link between smoking history and the risk of advanced liver fibrosis at diagnosis in PBC. The mechanism by which smoking may accelerate the histological progression of $\mathrm{PBC}$ is unknown and larger studies are needed to define it.

Disclosure of Interest None Declared.

\section{PWE-133 HEPATOCELLULAR CARCINOMA: IMPACT OF NICE GUIDELINES ON ASSESSING AND OPTIMISING SURVEILLANCE}

E Johnston*, R Khan, J Ingoe, G Tritto. Gastroenterology, Guy's and St Thomas' NHS Foundation Trust, London, UK

\subsection{6/gutjnl-2014-307263.393}

Introduction Screening for hepatocellular carcinoma (HCC) is only deemed cost-effective in patients at high risk. However, the criteria for identifying patients at risk, particularly in relation to non-cirrhotic hepatitis B, remains ill defined and different international guidelines are not consistent. Recent NICE guidelines proposed offering surveillance to patients with significant fibrosis $(\geq \mathrm{F} 2)$ or age $>40$ with either high viral load or family history of HCC. The aim of this study was to evaluate how the new NICE guidelines can impact on current practice and on the effectiveness of our surveillance program.

Methods All patients who underwent an abdominal ultrasound for HCC surveillance from $1^{\text {st }}$ September 2012-31 $1^{\text {st }}$ August 2013 were identified and the indications evaluated. In a subgroup of these patients with hepatitis B the appropriateness of screening was assessed according to the 2013 NICE guidelines. From the same period, all HCC diagnosed were identified and cross matched with our surveillance list.

Results In the period under study, 1280 surveillance ultrasounds (606 in cirrhosis, 628 in non cirrhotic hepatitis B or C, 46 other) led to 5 diagnoses of HCC. All HCC were found in cirrhotic patients (annual incidence 1.65\%). Seventeen further HCC were diagnosed from emergency admissions or referred from elsewhere. One patient had non cirrhotic hepatitis B (78yr old male with a long history of hepatitis $B$ ) whilst all the remaining were cirrhotic (2 with hepatitis B). In a randomly selected sample of 200 patients undergoing surveillance ultrasounds, $62 \%(n=124)$ had hepatitis B and 76 had cirrhosis of other aetiology. Of the patients with hepatitis $B$, an assessment of specific risk factors, particularly level of fibrosis, was not documented in 50 (40\%). A further 48 (39\%) patients were retrospectively deemed low risk according to NICE guidance.

Conclusion The number of HCC diagnosed through surveillance was low, but still cost effective in cirrhotic patients. The main risk factor for HCC in this cohort was cirrhosis (96.5\%). In fact, only 1 non cirrhotic patient with hepatitis B developed HCC and this was on a background of likely advanced fibrosis. None of the HCC from surveillance would have been missed if NICE criteria were used. It is important that patients with chronic hepatitis $\mathrm{B}$ all undergo assessment of fibrosis, along with clear documentation of other known risk factors in order to enable better risk stratification. In our experience following NICE guidance to identify high risk patients for surveillance will pick up all cases of HCC and decrease cost by reducing the number of unnecessary ultrasound scans being carried out.

\section{REFERENCE}

NICE clinical guideline 165 - Hepatitis B (chronic) June 2013 (http://www.nice.org.uk/ nicemedia/live/14191/64234/64234.pdf)

Disclosure of Interest None Declared.

\section{PWE-134 OVERVIEW OF EFFICACY AND COST EFFECTIVENESS OF NURSE LED DAY CASE ABDOMINAL PARACENTESIS SERVICE AT GLOUCESTERSHIRE HOSPITAL NHS FOUNDATION TRUST}

F Tahir*, C Hollywood, D Durrant. Gastroenterology Department, Gloucestershire NHS Foundation Trust, Gloucester, UK

\subsection{6/gutjnl-2014-307263.394}

Introduction Liver disease is the fifth most common cause of death in England and Wales ${ }^{1}$ and is one of the important causes of acute hospital admissions. Ascites is a common complication of Cirrhosis and patient with refractory ascites often require therapeutic paracentesis for symptomatic relief. In majority of UK hospitals, therapeutic paracentesis is done via planned admission to an acute medical bed which often causes delay waiting for a bed. In one study, the average hospital stay for therapeutic paracentesis was 5.5 days, ${ }^{2}$ as they have to wait for abdominal ultrasound to mark a suitable drain site. This not only leads to patient dissatisfaction but also has significant cost implications for the Trust.

Methods Retrospect patient's data from 2010-2013 was reviewed who attended our Ambulatory Day Unit for therapeutic paracentesis.

Results The service was set up in 2010 at the Gloucestershire Royal Hospitals with a view to perform 1-2 paracentesis weekly, which then gradually expanded to up to12-14 paracentesis week. Paracentesis was performed by an experienced and dedicated liver specialist nurse, using aseptic technique and under Human Albumin solution (100 mls of 20\%) cover for every 3 litres of ascites drained. 111 patients have so far benefited from the service and 764 procedures are performed with 6 failed attempts in a single patient only with malignant ascites. There have been no complications. $63(56.7 \%)$ patients required paracentesis once every two or more months whilst 19 (17.1\%) needed weekly drains. The most number of attendances by a single patient was 62 . The day case service is cost effective and has saved the Trust over $£ 30,000$ per annum. Patients love this service as it is easily accessible and has significantly reduced the waiting time for paracentesis as the service is available to patients within a few days and they are able to self-refer.

Conclusion We found the nurse led day case therapeutic paracentesis service is safe and cost effective. It is easily accessible and is liked by the patients as it gives them more control on their treatment and better continuity of their care. It has also a potential to provide training opportunities to junior doctors. We advocate setting up similar services in other NHS Trusts

\section{REFERENCES}

1 The Health Survey for England 2007

2 Hill $S$, et al. Developing a nurse led, day-case, abdominal paracentesis service. Cancer Nursing Practice June 2013;12(5)

Disclosure of Interest None Declared. 